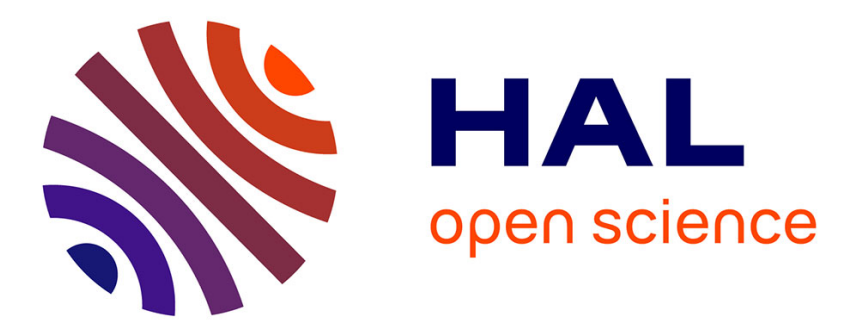

\title{
Parameters governing strain induced crystallization in filled natural rubber
}

\author{
J.-M. Chenal, Catherine Gauthier, Laurent Chazeau, Laurent Guy, Yves
} Bomal

\section{- To cite this version:}

J.-M. Chenal, Catherine Gauthier, Laurent Chazeau, Laurent Guy, Yves Bomal. Parameters governing strain induced crystallization in filled natural rubber. Polymer, 2007, pp.6893-6901. 10.1016/j.polymer.2007.09.023 . hal-00434168

\section{HAL Id: hal-00434168 \\ https://hal.science/hal-00434168}

Submitted on 19 Sep 2018

HAL is a multi-disciplinary open access archive for the deposit and dissemination of scientific research documents, whether they are published or not. The documents may come from teaching and research institutions in France or abroad, or from public or private research centers.
L'archive ouverte pluridisciplinaire HAL, est destinée au dépôt et à la diffusion de documents scientifiques de niveau recherche, publiés ou non, émanant des établissements d'enseignement et de recherche français ou étrangers, des laboratoires publics ou privés. 


\title{
Parameters governing strain induced crystallization in filled natural rubber
}

\author{
Jean-Marc Chenal $^{\mathrm{a}, *}$, Catherine Gauthier ${ }^{\mathrm{a}}$, Laurent Chazeau ${ }^{\mathrm{a}}$, Laurent Guy ${ }^{\mathrm{b}}$, Yves Bomal ${ }^{\mathrm{b}}$ \\ ${ }^{a}$ MATEIS, Bâtiment Blaise Pascal, INSA-Lyon, 20, Avenue Albert Einstein, F-69621 Villeurbanne Cedex, France \\ ${ }^{\mathrm{b}}$ Rhodia Recherches et Technologie, CRTL, 85 rue des frères Perret, 69192 St Fons Cedex, France
}

\begin{abstract}
A series of in situ synchrotron X-ray diffraction experiments are performed during the stretching of weakly and highly vulcanized carbon black (CB), silica and grafted silica filled natural rubber sample (NR). Conversely to literature, Mullins effect observed after one stretching cycle modifies the strain induced crystallization (SIC) behaviour of the sample. The onset of crystallization is ruled by the strain amplification induced by the filler presence. Moreover, fillers (CB and silica) behave as additional crosslinks into NR network, through filler-rubber interactions that either accelerate or slow down the crystallization rate depending on NR matrix chemical crosslink density. This is consistent with the assumption that effective network density, which is due to chemical crosslinks, entanglements, and filler-rubber interactions, controls the crystallization rate.
\end{abstract}

Keywords: Natural rubber; WAXD; Carbon black and silica

\section{Introduction}

Reinforcement of rubber by fillers has been the topic of abundant literature [1,2]. The effect of filler can be analysed at two levels. On a macroscopic level, it is well established that the introduction of specific filler such as carbon black and silica in rubber can strongly increase its rigidity, its abrasion resistance, tear strength and stress at break. These improvements are the result of a complex interplay between the properties of the individual constituent phases (the polymer and the filler) and the filler-filler and filler-rubber interactions. Thus, filler type, volume fraction, specific surface, structure and surface chemical treatment have a large influence on the mechanical behaviour of the material.

Among various types of elastomers, natural rubber (NR) is one of the most used in industrial and household applications [3]. The interest of NR is mainly due to its self-reinforcement character, which is said to originate from its strain induced crystallization (hereafter referred as SIC). The introduction

\footnotetext{
* Corresponding author. Tel.: +3347243 6129; fax: +33472438528. E-mail address: Jean-Marc.Chenal@insa-lyon.fr (J.-M. Chenal).
}

of filler in NR changes the stress field, increases the local strain of the chain (also called strain amplification), and leads to local heterogeneities. Thus, one key problem is to understand the influence of the filler, like carbon black (CB) and silica filler, on the crystallization properties of the NR rubber matrix.

Some studies [4,5] demonstrated that carbon blacks increase the amount of strain induced crystallization in the crack tip region, and suggested that this contributes significantly to crack growth resistance. Recently, Trabelsi et al. [6], Poompradub et al. [7] and Rault et al. [8] investigated the effective local deformation in stretched CB filled NR by in situ synchrotron X-ray measurements during uniaxial deformation. They demonstrated that the strain at the crystallization onset decreases with the addition of filler, due to strain amplification.

Another aspect investigated by Poompradub et al. [7] and Trabelsi et al. [6] using WAXS was the evolution of the crystallite sizes versus strain in the case of CB filled NR. Poompradub et al. have measured the crystallites' dimensions perpendicular $\left(L_{200}\right.$ and $\left.L_{120}\right)$ to the stretching direction while Trabelsi et al. only determined the one parallel $\left(L_{002}\right)$ to it. In each study, it was shown that when fillers are introduced, the crystallite size is decreased like the degree of crystallite 
orientation. However, in a previous study, Gehman [9] has reported that the strain induced crystallites in a CB filled vulcanized NR have the same size as those in the deformed neat gum. In each of these studies, note that the SIC properties of carbon black filled NR have always been established for one type of carbon black at one NR crosslink density. Moreover, in spite of the importance of silica filled NR in industrial applications, the SIC of silica filled NR has never been reported.

Thus, the aim of our study is to clarify the influence of filler characteristics on the SIC properties (i.e. crystallinity, onset and rate of crystallization, crystallites' size). We compare the behaviour of samples processed with various types of filler for two different NR matrix crosslink densities, also known to strongly influence the SIC [10]. Three types of carbon black are used with different specific surfaces (and the same structure) and with different structures (and the same specific surface). In addition, one type of silica with different surface chemistries (grafted or ungrafted) is also studied. The SIC properties of the different samples are studied, thanks to in situ synchrotron X-ray diffraction measurements (WAXS), to characterize both crystallization kinetics and morphology (crystallites' sizes and orientation). Moreover, to estimate the decohesion (filler-filler and filler-matrix) and the cavitation (NR matrix) phenomena during stretching, volume change measurements during deformation are performed, thanks to a specific apparatus developed in the laboratory.

\section{Experimental section}

\subsection{Materials}

Natural rubber (SMR 10), three CB with different structures and specific surfaces (N234, N347, N330 from Cabot) and silica $\left(\mathrm{Z} 1165 \mathrm{MP}^{\circledR}\right.$ supplied by Rhodia Recherches et Technologie) are used in this study. The specific surface and structure determined by DBPA crushed method are reported in Table 1. Silica is grafted either with methyltrimethoxysilane and octyltrimethoxysilane (hereafter referred as covering agent: AR1 and AR8) or bis(triethoxysilylpropyl)tetrasulfide (hereafter referred as coupling agent: AC or TESPT), in order to modify filler-filler and filler-rubber interactions. Grafting is carried out in situ during processing and the surface grafting density is evaluated following the method described by

Table 1

Physical properties of fillers

\begin{tabular}{llll}
\hline Filler & $\begin{array}{l}\text { Average particle } \\
\text { size }(\mathrm{nm})\end{array}$ & $\begin{array}{l}\text { Surface area } \\
\left(\mathrm{m}^{2} / \mathrm{g}\right)\end{array}$ & $\begin{array}{l}\text { DBPA crushed } \\
(\mathrm{ml} / 100 \mathrm{~g})\end{array}$ \\
\hline N234 & $24-29$ & 124 & 101 \\
N347 & $30-35$ & 87 & 100 \\
N330 & $28-36$ & 84 & 90 \\
Z1165MP & $9-10$ & 160 & -
\end{tabular}

${ }^{\text {a }}$ DBPA (Dibutylphthalate adsorption) crushed method (ASTM D-3493) is a practical way of determining structure by measuring the void volume after several compressions of fillers in order to take into account the break-up of filler structure during compounding.
Ramier et al. [11]. In both cases (with AR and AC), the same surface grafting density is reached.

Unfilled and filled NR samples (supplied by Rhodia Recherches et Technologie) have been obtained by sulfur vulcanization of natural rubber according to the recipes given in Tables 2 and 3. Filler loadings are expressed in phr (grams of filler per $100 \mathrm{~g}$ of rubber). The filler volume ratio is equal to $20 \%$ (this corresponds to $45 \mathrm{phr}$ of carbon black or $50 \mathrm{phr}$ of silica). Samples are vulcanized either with chemical crosslink density higher or lower than the physical crosslink density in $\operatorname{NR}\left(\nu_{\mathrm{e}} \sim 1.3 \times 10^{-4} \mathrm{~mol} \mathrm{~cm}{ }^{-3}\right)$ [12]. This is obtained by changing the accelerator (CBS) concentration. The cure time $\left(t_{98}\right.$ at $150{ }^{\circ} \mathrm{C}$ ) is determined from torque measurement as a function of temperature performed with a Monsanto analyser. Matrix network chains' density $\left(\nu_{\mathrm{c}}\right)$, presented in Tables 2 and 3 , has been determined from swelling ratio in toluene $\left(Q_{\mathrm{pol}}=V / V_{0}\right.$, where $V$ and $V_{0}$ are the volumes of the polymer network, respectively, at swelling equilibrium and after swelling and drying) and Flory-Rehner equation [13]:

$-\left[\ln \left(1-v_{2}\right)+v_{2}+\chi_{1} v_{2}^{2}\right]=V_{1} \nu_{\mathrm{c}}\left[v_{2}^{1 / 3}-\frac{v_{2}}{2}\right]$

where $v_{2}$ (or $1 / Q_{\mathrm{pol}}$ ) is the volume fraction of polymer in the swollen mass, $V_{1}\left(106.3 \mathrm{~cm}^{3} / \mathrm{mol}\right)$ is the molar volume of the solvent (toluene), $\nu_{c}$ represents the number of active network chains per unit volume and $\chi_{1}$ is Flory-Huggins polymer-solvent dimensionless interaction term $\left(\chi_{1}\right.$ is equal to 0.39 for the NR-toluene system [14]).

The swelling procedure was applied to the filled vulcanizates in order to evaluate the number of filler-rubber bonds. In the case of unfilled NR, the swelling restriction is due to crosslinks connecting the polymer chains, which avoid their extension and their diffusion. The addition of filler is equivalent to the introduction of additional crosslinks due to the filler-rubber interactions. With the assumption of a perfect interface and as a first approximation, the relation between the

Table 2

Compositions of carbon black/SMR 10 rubber composites and corresponding vulcanized unfilled NR matrices

\begin{tabular}{lllllll}
\hline Sample code & NR-1 & S-1 & S-2 & S-3 & NR-2 & S-4 \\
\hline NR (SMR 10 $)$, phr & 100 & 100 & 100 & 100 & 100 & 100 \\
N234, phr & & 45 & & & & 45 \\
N347, phr & & & 45 & & & \\
N330, phr & 2 & 2 & 2 & 2 & 0 & 0 \\
Stearic acid, phr & 4 & 4 & 4 & 4 & 0 & 0 \\
ZnO, phr & 1.5 & 1.5 & 1.5 & 1.5 & 0 & 0 \\
6PPD, phr & 1.75 & 1.75 & 1.75 & 1.75 & 0 & 0 \\
CBS ${ }^{b}$ phr & 1.5 & 1.5 & 1.5 & 1.5 & 1.5 & 1.5 \\
Sulfur, phr & 4.65 & 4.04 & 4.00 & 3.97 & 11.61 & 7.16 \\
$Q_{\text {pol }}( \pm 1 \%)$ & 1.71 & 2.41 & 2.47 & 2.37 & 0.25 & 0.65 \\
Network chain density & & & & & & \\
$\quad($ swelling $\nu) \times 10^{4} \mathrm{~mol}^{\mathrm{c}} / \mathrm{cm}^{3}$ & & & & 45 & & \\
\hline
\end{tabular}

\footnotetext{
a Standard Malaysian Rubber number 10.

b $N$-Cyclohexyl-2-benzothiazole sulfenamide.

c Network chain density determined from swelling ratio of the polymer itself.
} 
Table 3

Compositions of silica/SMR 10 rubber composites and corresponding vulcanized unfilled NR matrices

\begin{tabular}{llllllll}
\hline Sample code & NR-2 & S-5 & S-6 & S-7 & S-8 & NR-3 & S-9 \\
\hline NR (SMR 10), phr & 100 & 100 & 100 & 100 & 100 & 100 & 100 \\
Z1165, phr & & 50 & 50 & 50 & 50 & & 50 \\
AR1, phr & & & 1.4 & & & & \\
AR8 & & & & 2.1 & & & \\
TESPT, phr & 0 & 0 & 0 & 0 & 0 & 2 & 2 \\
Stearic acid, phr & 0 & 0 & 0 & 0 & 0 & 4 & 4 \\
ZnO, phr & 0 & 0 & 0 & 0 & 0 & 1.5 & 1.5 \\
6PPD, phr & 0 & 0 & 0 & 0 & 0 & 2.5 & 2.5 \\
CBS, phr & 1.5 & 1.5 & 1.5 & 1.5 & 1.5 & 1.5 & 1.5 \\
Sulfur, phr & 11.61 & 6.98 & 7.21 & 7.71 & 5.36 & 4.4 & 4 \\
$Q_{\text {pol }}( \pm 1 \%)$ & 0.25 & 0.69 & 0.64 & 0.56 & 1.24 & 1.91 & 2.47 \\
Network chain density & & & & & & & \\
$\quad($ swelling $\nu) \times 10^{4} \mathrm{~mol} / \mathrm{cm}^{3}$ & & & & 4 & & 4 \\
\hline
\end{tabular}

swelling ratio of the polymer itself $\left(Q_{\mathrm{pol}}\right)$ and the equilibrium swelling ratio of the composite $(Q)$ is deduced from the formula:

$Q_{\mathrm{pol}}=\frac{Q-\varphi_{\mathrm{f}}}{1-\varphi_{\mathrm{f}}}$

where $\varphi_{\mathrm{f}}$ is the filler volume fraction.

The rubber swelling ratio of the compounded filled samples is always found lower than that of the neat NR matrix (Tables 2 and 3). Indeed, this restriction comes either from the strong adsorption of the matrix on the filler surface and/or from a modification of the crosslink density in the bulk. From a phenomenological point of view, Rault et al. [8] have proposed that the effective crosslink density $(\nu)$ deduced from this swelling value can be seen as the sum of the chemical crosslinks $\left(\nu_{\mathrm{c}} \sim 1 / M_{\mathrm{c}}\right)$, entanglements $\left(\nu_{\mathrm{e}} \sim 1 / M_{\mathrm{e}}\right)$ and elastomer chains' adsorption on the filler $\left(\nu_{\mathrm{G}} \sim 1 / M_{\mathrm{G}}\right)$ densities. The influence of the filler presence on the vulcanization has been previously studied. Wolff [15] demonstrated that CB has no significant influence on the vulcanization process. As silica filled NR vulcanization can be perturbed by the adsorption of CBS on the silica surface, then, most of silica samples have been vulcanized without CBS (S-5, S-6, S-7 and S-8). In the case of silica filled sample (S-9), the effect of CBS on the crosslink density can be neglected with regard to the additional chemical crosslinks due to sulfur release of the coupling agent. The effective crosslink densities calculated from swelling measurements (Table 3) confirm the effect of each grafting agent: AR agent enables to decrease filler-matrix interactions, conversely to $\mathrm{AC}$ agent which promotes covalent bonds between the matrix and the filler. In addition, by taking into account the study of Sajjayanukul et al. [16] who prepared CB filled NR with a similar protocol to ours, we can disregard the effect of CB dispersion, because they showed that the type of carbon black $(\mathrm{N} 220, \ldots, \mathrm{N} 660)$ induced no significant difference in degree of carbon black dispersion in NR matrix. In the case of silica $\left(\mathrm{Z} 1165 \mathrm{MP}^{\circledR}\right)$ Ramier et al. [17] demonstrated that silica dispersion was the same whatever the type and the amount of grafting agent.

\subsection{Instruments}

\subsubsection{Tensile tests}

Stress-strain measurements were carried out by stretching dumbbell shaped specimens. The initial length, width and thickness of the parallel part of the dumbbell were $9 \times 3 \times 1 \mathrm{~mm}^{3}$. The tests were performed at room temperature and $0.25 \mathrm{~min}^{-1}$.

\subsubsection{Volume change measurement during stretching}

Volume change measurements were performed using a specific apparatus developed in the laboratory based on Farris apparatus. The equipment is based on the following principle: a specimen chamber and a reference chamber filled with gas are connected by a differential pressure sensor. When the sample is stretched, a volume change of the sample causes a pressure change relative to the reference chamber. Via the known volumes of sample and sample chamber, the pressure difference can be converted into a volume change of the sample. A pressure difference will also be recorded if the sample adsorbs gas from the surroundings during deformation [18]. In order to avoid this phenomenon, argon (Ar), which is not absorbed by samples, was chosen to fill specimen and reference chambers.

\subsubsection{In situ WAXS measurement during stretching}

A homemade stretching machine allowing the symmetric deformation of the sample was used to probe by X-ray the same sample area during stretching at $0.25 \mathrm{~min}^{-1}$ strain rate. High resolution is necessary for real-time measurements. Thus in situ wide-angle X-ray scattering (WAXS) study was carried out on the D2AM beamline of the European Synchrotron Radiation Facility (ESRF). The X-ray wavelength is $0.54 \AA$. The two-dimensional (2D) WAXS patterns were recorded every $10 \mathrm{~s}$ by a CCD Camera (Princeton Instrument). During stretching, the thickness and then the absorption of the sample decrease. Therefore, the in situ measurements of the absorption by photomultiplicators, located ahead and behind the sample, are used to normalize the scattered intensities.

The crystal structure of natural rubber is orthorhombic and its space group is $\mathrm{Pbca}$ [19]. Thus, the crystallite orientation along the draw axis is deduced from the intensity distribution of the 002 reflection, which is obtained by the azimuthal scan of the peak. Experimental reflection profile is fitted with Gaussian function and can be fully characterized by its halfwidth at half-height $\left(\psi_{1 / 2}\right)$. In this work, the crystallinity $\left(X_{\mathrm{c}}\right)$ is determined using the simplified method of Mitchell [20], which has been applied in several studies. The average crystallites' sizes $L_{h k l}$ in the direction normal to the $(h k l)$ planes are estimated from Scherrer equation:

$L_{h k l}=K \lambda /\left(\beta_{1 / 2} \cos \theta\right)$

where $\lambda$ is the wavelength and $\theta$ is Bragg angle. Each peak (200, 120 and 002) is fitted with a Lorentzian in which the half-width at half-height is $\beta_{1 / 2}$. According to the parameters chosen for the simulation of experimental peaks, the value selected for $K$ is 0.64 . It is worth noting that the variation of the three crystallites' dimensions is simultaneously measured 
during the stretching that gives access to the mean crystallites' volume. In the other previous dynamic studies of strain induced crystallization $[6,7]$ the crystallites' mean volume could not be measured.

\section{Results and discussion}

\subsection{Stress induced crystallization and Mullins effect}

In order to understand the impact of Mullins effect on SIC, we have measured simultaneously the tensile stress, the volume change and the crystallinity of the different samples. Results are illustrated in the case of S-1 sample during three successive cycles at $T=20{ }^{\circ} \mathrm{C}$ and $0.25 \mathrm{~min}^{-1}$ strain rate as a function of the draw ratio (Figs. 1-3). Considering Fig. 1a, Mullins softening may be defined as the reduction of stress caused by successive stretching. The main drop of the stress is observed after the first stretching cycle and one can consider that the stress is "stabilized" after the third cycle. This phenomenon is characteristic of reinforcing filler (silica, carbon black), but occurs to a lesser extent in gum. Explanations of Mullins effect have included breakage of weak linkages, of network chains extending between adjacent filler particles, and of polymer-filler bond [21]. As shown in Fig. 1b, during the stretching process of S-1 sample, a positive volume variation, which is mainly due to filler-rubber decohesion and matrix cavitation, is recorded. Like Mullins effect, the positive volume variation decreases after successive cycles until stabilization. During the retraction process, a fast recovery of decohesion/cavitation is observed. Moreover, the presence of a negative volume variation recovered with a longer timescale enables to highlight the strain induced crystallization of NR. Fig. 2 schemes the different phenomena (decohesion/cavitation and crystallization) evidenced by volume variation measurements. A similar behaviour has been observed with all the samples. However, due to the choice of the pressure sensor, accuracy was not high enough to measure crystallinity differences between our filled samples.

To do so, we have also performed in situ WAXS measurements on the same samples. Results obtained with S-1 are shown in Fig. 3. Between the first and the other cycles, the crystallinity evolution is significantly different. The onset of crystallinity shifts toward higher draw ratio and the crystallinity values decrease. This result seems in disagreement with the one of Trabelsi et al. [6]. Indeed, they have concluded that Mullins effect does not play any role during SIC (stretching) and during melting (recovery). However, it is worth noting that Trabelsi experiments have been performed at $0.033 \mathrm{~min}^{-1}$ while our measurements have been performed at $0.25 \mathrm{~min}^{-1}$. Boonstra [21] has suggested that upon initial stretching of the sample, molecular slippage mechanisms operate at the fillerrubber interface, or some chains are broken to redistribute the stress. This process is highly rate dependent. The discrepancy between our experiments and those of Trabelsi is an indication that at $0.033 \mathrm{~min}^{-1}$ the stress in the matrix network is sufficiently low to avoid any important modification of the rubber-filler network and consequently of SIC. (a)

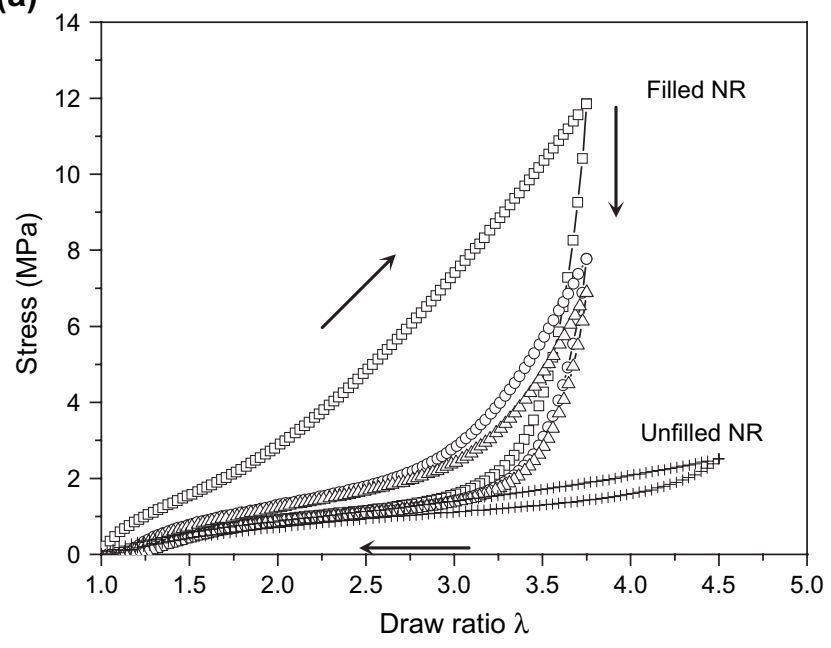

(b)

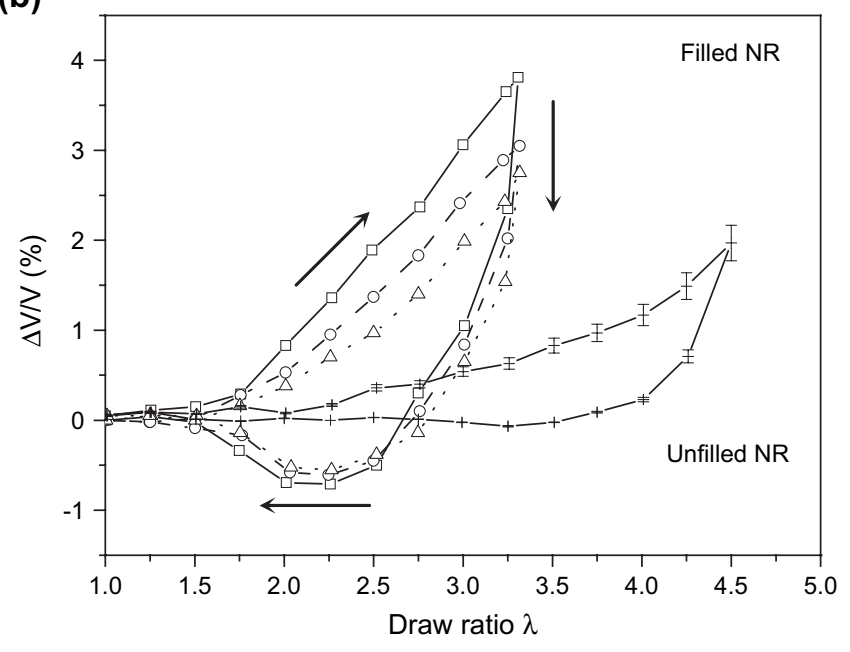

Fig. 1. (a) Stress-strain curves and (b) volume change-strain curves of filled and unfilled NR measured during mechanical cycles at $T=20^{\circ} \mathrm{C}$, strain rate $0.25 \mathrm{~min}^{-1}:(\square) 1$ st cycle, $(\bigcirc) 2$ nd cycle, $(\triangle)$ 3rd cycle.

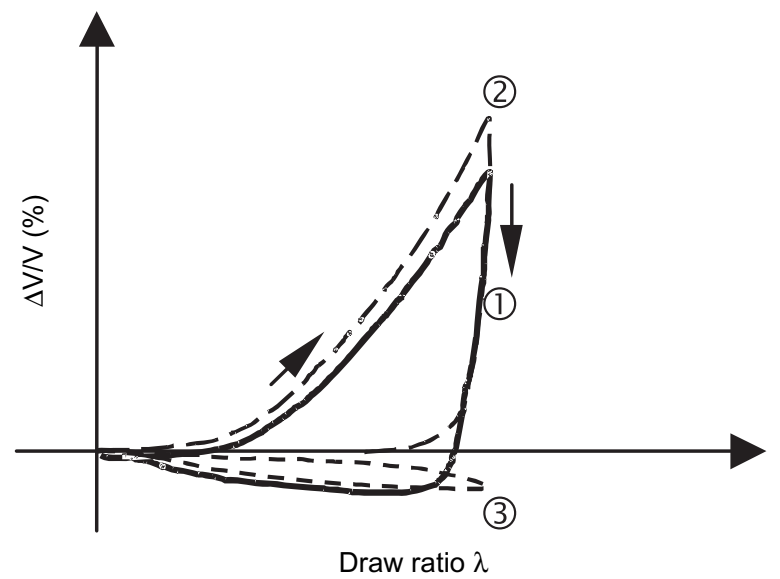

Fig. 2. Theoretical curve of volume change versus strain (1) depending on cavitation/decohesion (2) and crystallization (3). 


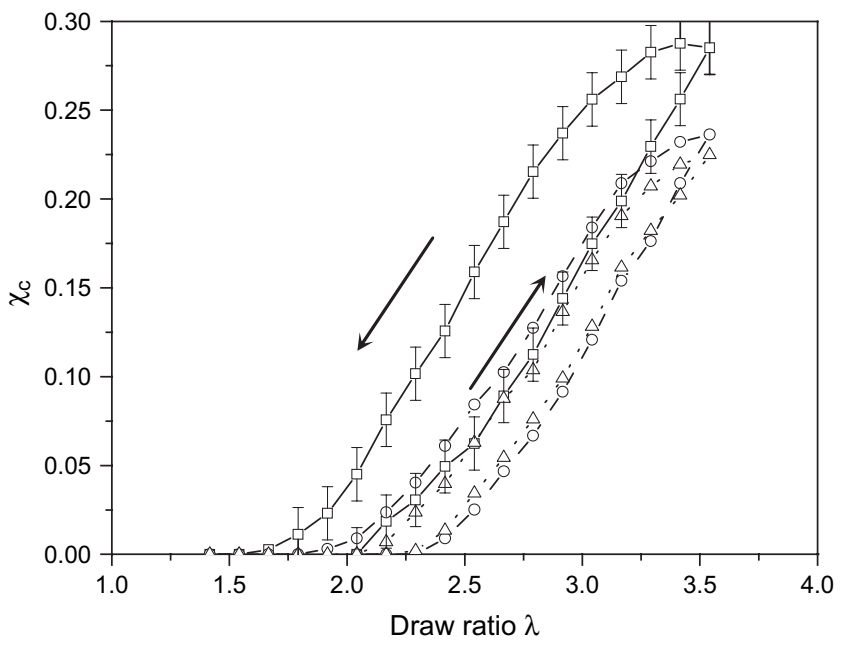

Fig. 3. Change of crystallinity during successive cycles as a function of draw ratio: $(\square)$ 1st cycle, $(\bigcirc)$ 2nd cycle, $(\triangle)$ 3rd cycle.

In the following, WAXS results concern the third stretching, i.e. what we called "demullinized" samples.

\subsection{Effect of crosslink density on SIC of CB filled NR}

\subsubsection{Tensile properties}

Tensile stress-strain curves of filled (with N234) and unfilled natural rubber samples are shown in Fig. 4a. A stress upturn is observed. For rubber materials, it is generally associated to the stress at which the smallest chains reach their critical extensibility. In the case of NR, this stress upturn is also associated to the onset of crystallization. For high and for low crosslink density matrices (NR-1 and NR-2), the stress upturn (deduced from the study of the curve derivative) is found at $\lambda$ around 3.5 and 4 , respectively. In the presence of filler, the stress upturn occurs at a same draw ratio, around 2 , for both S-1 and S-4 samples. This suggests that the onset of SIC in the case of filled samples is independent of the chemical crosslink density of the matrix. This result is not trivial and must be confirmed by the crystallinity study.

\subsubsection{Crystallinity}

The crystallinity $\left(\chi_{\mathrm{c}}\right)$ measured in situ by WAXS during uniaxial deformation for S-1 and S-4 samples and their corresponding matrix NR-1 and NR-2 is reported in Fig. 5. Crystallites' orientation has been determined and displays almost a constant average value versus draw ratio. Although filler promotes strain induced crystallization, orientation is poorer in the filled sample $\left(\psi_{1 / 2} \sim 10^{\circ}\right)$ than in the vulcanizated gum $\left(\psi_{1 / 2} \sim 5^{\circ}\right)$. This probably results from the perturbation of the stress field induced by the presence of rigid inclusions. In gum rubber, chains can relatively easily line-up uniaxially and crystallize, whereas in the presence of carbon black, the stress field is complex and crystallites may grow along complex stress contours.

The X-ray measurements show that the crystallites develop at $\lambda$ around 3.5 and 4 for NR-1 and NR-2, respectively. The draw ratio $(\lambda)$ is found around 2 for both $S-1$ and $S-4$ samples. (a)

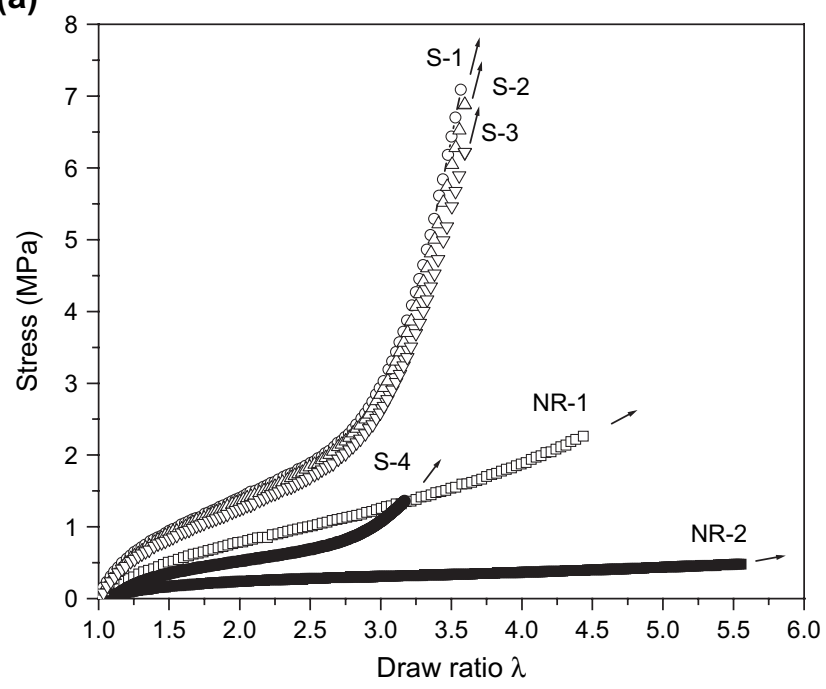

(b)

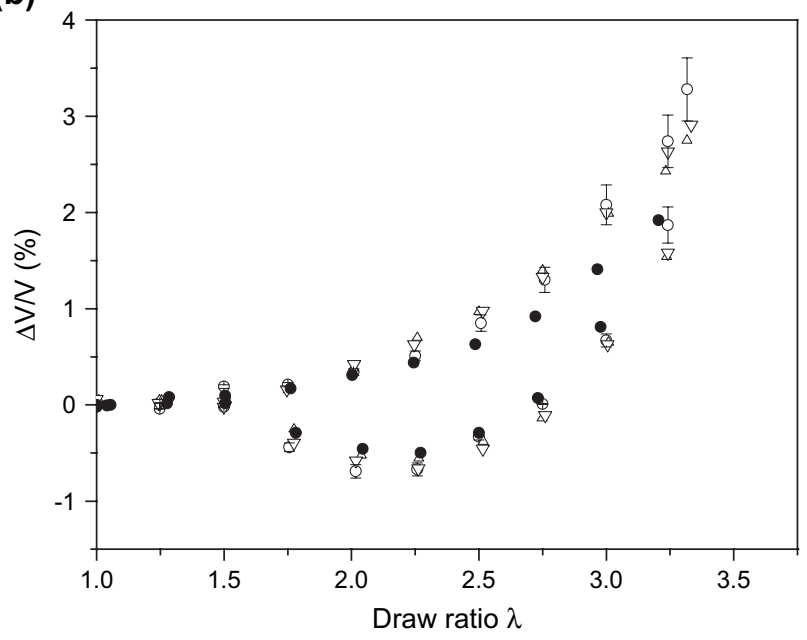

Fig. 4. (a) Stress-strain curve of demullinized samples at $T=20^{\circ} \mathrm{C}$, strain rate $0.25 \mathrm{~min}^{-1}$ : (O) $\mathrm{S}-1,(\triangle) \mathrm{S}-2,(\nabla) \mathrm{S}-3,(\bullet) \mathrm{S}-4$ and $(\square) \mathrm{NR}-1,($

NR-2. (b) Volume change-strain curves.

These values are the same as those found previously for the stress upturn. This indicates that the SIC onset can indeed be associated to the stress upturn.

In the case of non-crystallizing rubber, it is known that the decrease of the strain at the stress upturn with the introduction of filler is related to strain amplification. In the case of filled rubber, Nielsen [22] has proposed a simple model in which the filler particles are linked to the polymer in a series arrangement and a perfect adhesion is assumed between the two phases. The local draw ratio of the filled NR rubber matrix $\left(\lambda^{\prime}\right)$ is evaluated with the following equation:

$\lambda^{\prime}=\left[(\lambda-1) /\left(1-\varphi^{1 / 3}\right)\right]+1$

where $\lambda$ is the macroscopic draw ratio of the filled sample and $\varphi$ is the filler loading.

Thus this expression is used to replot the crystallinity evolution of S-1 and S-4 versus draw ratio assuming that the only 


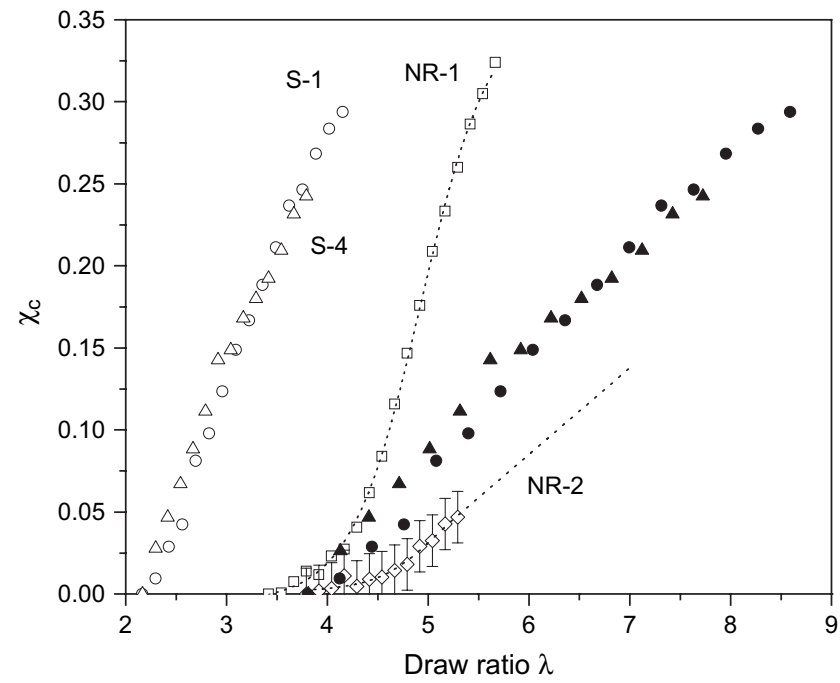

Fig. 5. Effect of matrix crosslink density on filled (N234) samples' crystallization (open symbols). Crystallinity versus NR filled matrix local draw ratio deduced from Nielsen equation (closed symbols).

effect of the filler is its strain amplification. The so-called "corrected S-1" and "corrected S-4" curves, displaying the crystallinity versus the rubber matrix local draw ratio, can be compared to the ones of NR-1 and NR-2 matrices, respectively (Fig. 5). The crystallization onset for "corrected S-1" and "corrected S-4" is remarkably close to each other and to those of NR-1 and NR-2. Given the uncertainties of the measurements for the very low crystallization fraction, it can be assumed that the crystallization onset in the samples is mainly ruled by the strain amplification, i.e. by the filler loading.

It is worth noting that the real strain rate of the filled rubber matrix is faster than the one applied to the composite $\left(0.25 \mathrm{~min}^{-1}\right)$. Consequently, we could expect a different crystallization rate for filled samples compared to the corresponding neat matrices. Qualitatively, crystallization rate can be evaluated from a linear extrapolation of $\chi_{\mathrm{c}}$ above 0.025 . It is well known that the NR crystallinity never reaches $100 \%$ either after cold crystallization or SIC. According to Gent [23], the higher the NR matrix chemical crosslink density, the lower the maximum crystallinity. In a previous paper, we [24] demonstrated that the NR cold crystallization (i.e. the optimum crystallinity) is much less sensitive to the introduction of filler (20\% v/v carbon black or silica) than the chemical crosslink density. Consequently, our interpretation is based on the comparison between filled and unfilled sample with the same NR matrix chemical crosslink density i.e. with a same optimum crystallinity.

\subsection{Effect of CB filler physical properties on SIC of filled $N R$}

In the case of filled samples, one can consider data corrected from matrix strain amplification. It is noticeable in Fig. 5 that both corrected S-1 and corrected S-4 samples have the same crystallization rate. However, the filled sample with the lower crosslink density matrix (corrected S-4) displays a small increase of the crystallization rate compared with the NR-2 matrix. The opposite phenomenon is observed in the case of the filled sample with the higher crosslink density matrix (S-1) when compared to the NR-1 matrix. Besides, in a general viewpoint, the increase of crystallinity could be due to either the increase of crystallites' numbers or of the size of primary crystallites or even to both.

To go further, size measurements in the three directions have been performed for unfilled matrices (NR-1 and NR-2) and filled samples (S-1 and S-4) versus crystallinity (plotted in Figs. 6 and 7, respectively). It clearly appears from Fig. 6 that the matrix with the higher crosslink density always shows the smaller crystallite dimensions. On the other hand, regardless of the matrix crosslink density, both $\mathrm{CB}$ filled samples have the same crystallites' sizes, close to that obtained for NR-1, Fig. 7. Note that, in this case, it is not possible to measure with enough precision the crystallite size $\left(L_{002}\right)$ below $10 \%$ of crystallinity because of the presence of CB filler amorphous halo on X-ray pattern. Above $10 \%$ of crystallinity for filled and unfilled samples, the crystallite sizes stay unchanged, which means that the crystallinity grows up during the stretching by the increase of the crystallites' number. Within this frame, the difference between NR-2 and S-4 should be due to a higher nucleation in the presence of fillers, associated to an increase of the effective crosslink density. Conversely, in the case of the highly crosslinked matrix, the results, i.e. slowing down of crystallization rate with $\mathrm{CB}$ and identical sizes and crystallization rates for $\mathrm{S}-1$ and $\mathrm{S}-4$, suggest that the introduction of CB in NR-1 matrix hinders the nucleation. The only convincing explanation is that the effective crosslink density becomes too high to allow nucleation in the vicinity of the filler (in the given experimental conditions). It is worth noting that the similarity between the crystallization rates of S-4 and S-1 is fortuitous. In the following, results for other filled samples will be analysed to confirm this interpretation.

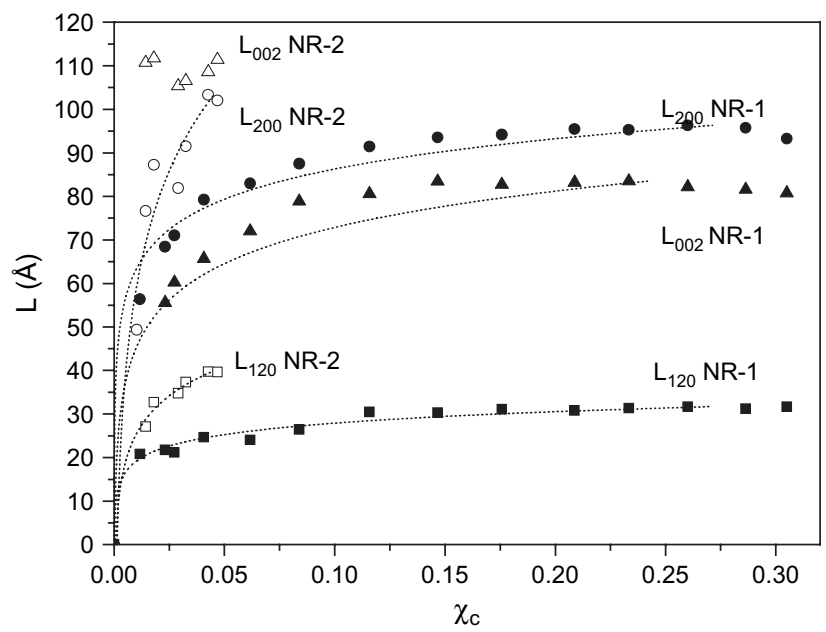

Fig. 6. Variation of crystallites' sizes $(\bigcirc, \bullet) L_{200},(\square, \boldsymbol{\square}) L_{120},(\triangle, \boldsymbol{\Delta}) L_{002}$ during the stretching process for each crosslinked matrix (NR-1: closed symbols and NR-2: open symbols) versus crystallinity. 


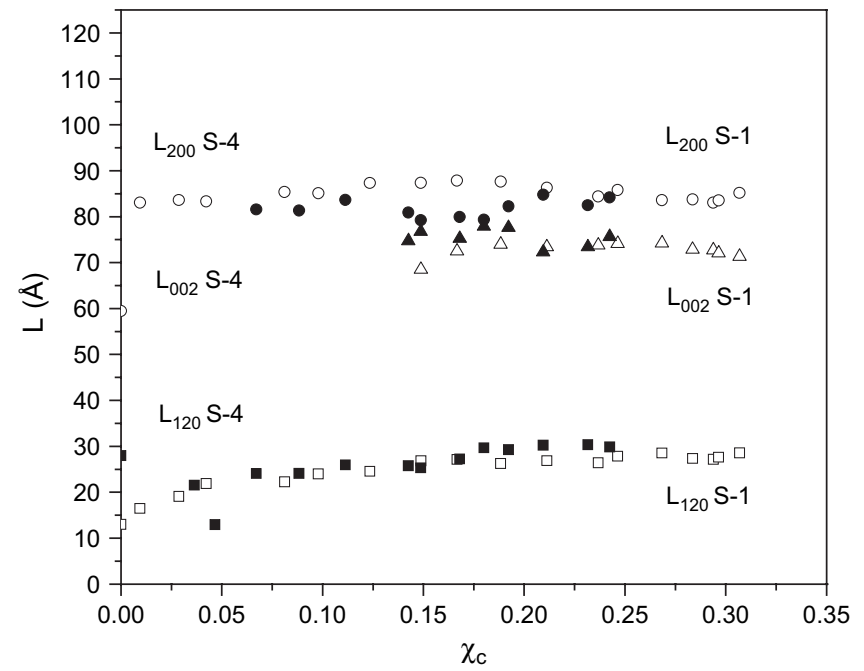

Fig. 7. Variation of crystallites' sizes $(\bigcirc, \boldsymbol{\bullet}) L_{200},(\square, \boldsymbol{\square}) L_{120},(\triangle, \boldsymbol{\Delta}) L_{002}$ during the stretching process for two samples filled with the same filler (S-1: closed symbols and S-4: open symbols) versus crystallinity.

The results of tensile stress-strain measurements of filled natural rubber sample with different kinds of $\mathrm{CB}$ filler are shown in Fig. 4a. All the samples (S-1, S-2, S-3) have a very similar behaviour, which is in agreement with the volume change measurements Fig. 4b, indicating that the three samples seem to reach the same levels of decohesion/cavitation and crystallization. Moreover, we note that the decrease of the crystallization onset with filler is similar for the three types of CB (for a given matrix crosslink density) (Fig. 8). However, it seems that the crystallization rate is slightly faster when the filler specific surface increases (S-1), but remains unchanged when the filler structure is modified (S-2, S-3). The three filled samples display the same evolution of crystallite sizes (unpresented data) and in each case the mean crystallite volume is identical $\left(200 \mathrm{~nm}^{3}\right)$. Thus, the effective crosslink density appears as a first order parameter and the filler specific surface as a second order parameter in the studied ranges. The

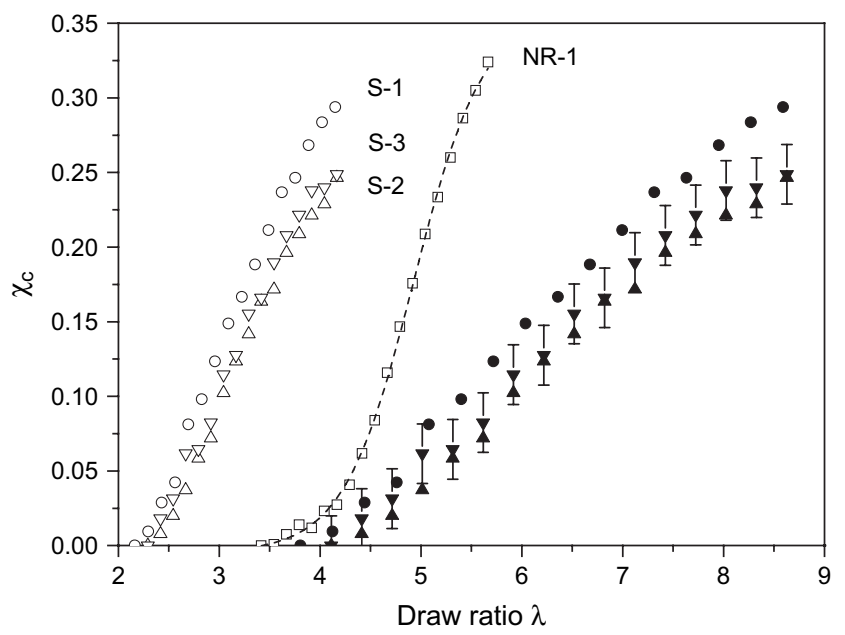

Fig. 8. Effect of CB properties on filled samples' crystallization (open symbols). Crystallinity versus NR filled matrix local draw ratio deduced from Nielsen equation (closed symbols). effective crosslink density might be a pertinent parameter to correlate to the crystallization rate (as defined previously). As shown in Fig. 9, it can be suggested that there is an optimum effective crosslink density for the crystallization rate. Additional data obtained with silica filled NR are required to address this question.

\subsection{Effect of silica filler on SIC of filled NR}

Tensile test measurements of filled natural rubber sample with different kinds of silica filler are displayed in Fig. 10. It clearly appears that the effect of AR grafting treatment (AR8 > AR1) decreases the mechanical properties contrarily to the effect of AC grafting treatment. Crystallinity versus draw ratio is plotted in Fig. 11a. The onset of crystallization is close to 2 for all samples. Again, the amplification phenomenon has been taken into account by plotting the corrected behaviour of the filled samples as a function of the draw ratio. The results confirm that the crystallization onset in the samples is governed by the strain amplification. Concerning size measurements, it appears like in the case of CB filled samples that a constant size is rapidly reached (unpresented data). From these measurements, the mean crystallite volume has been deduced. The values for S-5, S-6, S-7, S-8 and S-9 are, respectively, 325, 400, 425, 300 and $200 \mathrm{~nm}^{3}$. From these data, it appears that the volume decreases with increasing effective crosslink density (Fig. 12). Accordingly, the discussion of crystallization rate must take into account both the number of nuclei and their growth.

The crystallization rate of silica filled samples displays again two opposite trends according to crosslink density of the filled matrix, as shown in Fig. 9. Like in the case of CB filled NR, the introduction of silica in a weakly crosslinked matrix NR-2 slightly accelerates the NR crystallization rate (S-5, S-6, S-7, S-8 > NR-2). In any case, the crystallites' size for these four samples is smaller than that of NR-2, but the effective crosslink density is higher, leading to a higher

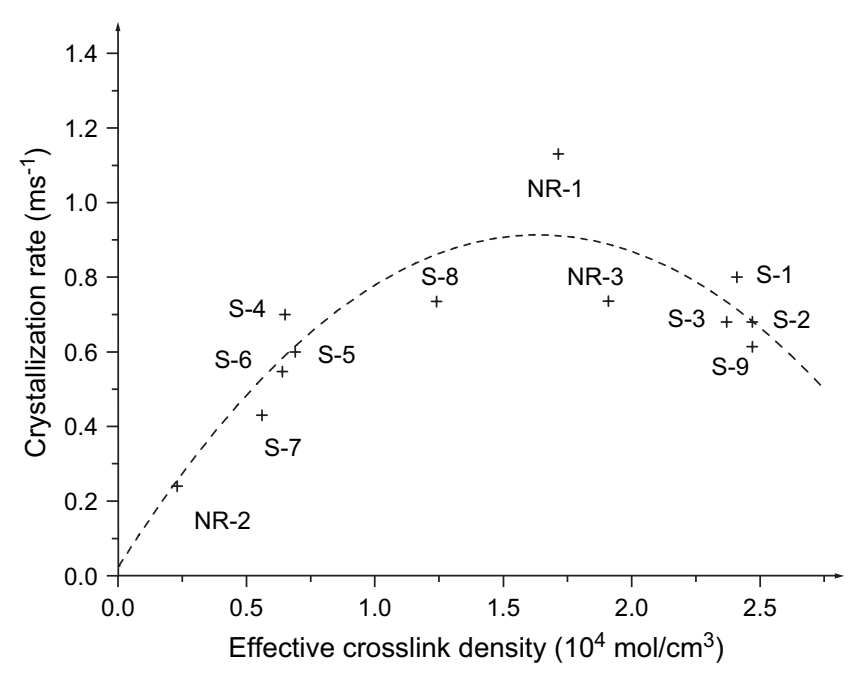

Fig. 9. Diagram displaying the crystallization rate versus the effective crosslink density for filled and unfilled samples. 


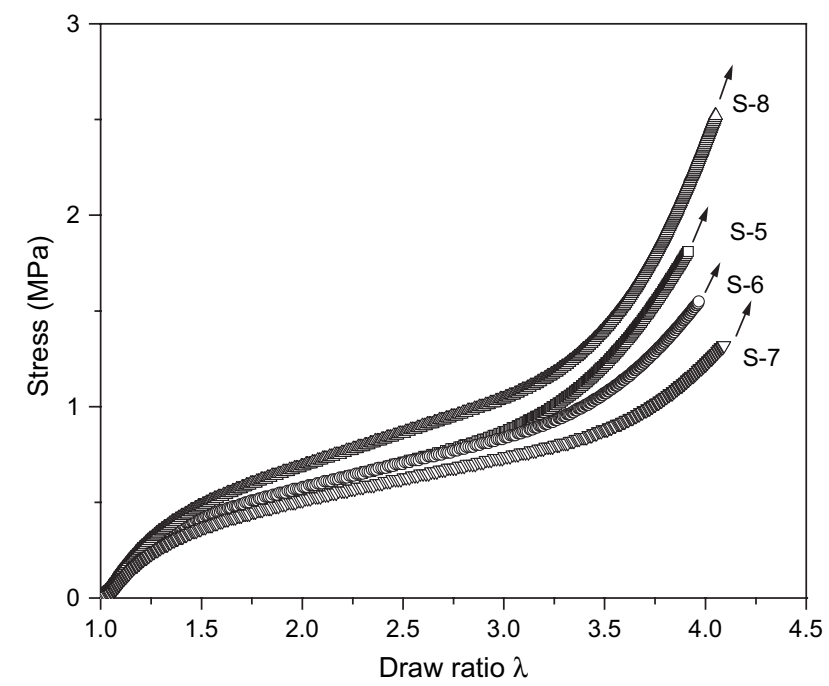

Fig. 10. Stress-strain curve of demullinized samples at $T=20^{\circ} \mathrm{C}$, strain rate $0.25 \mathrm{~min}^{-1}:(\square) \mathrm{S}-5,(\bigcirc) \mathrm{S}-6,(\nabla) \mathrm{S}-7,(\triangle) \mathrm{S}-8$.

nucleation rate. Among these samples, S-8 displays a peculiar behaviour. The crystallization rate is higher at the beginning of the SIC and then clearly decreases. The AC grafting treatment enables to increase notably the effective crosslink density by the chemical coupling silica-matrix and also via an additional vulcanization, thanks to TESPT (AC), sulfur release at high temperature. This is undoubtedly at the origin of the high initial crystallization rate. The decrease is assumed to be related to decohesion as indicated by volume variation measurements Fig. 11b. Note that, during stretching, the stress level increases faster in the case of S-8 (Fig. 10), that could accelerate the decohesion mechanism at a given draw ratio compared to S-5, S-6 and S-7.

On the other hand, the introduction of silica (more especially AC grafted silica: S-9) in a highly crosslinked matrix (NR-3) slows down the crystallization rate. The mean crystallite volume of S-9 sample is comparable to that of the corresponding unfilled matrix (NR-3). In that case, the decrease of the crystallization rate is due, as explained previously for S-1 compared to NR-1, to the effective crosslink density which becomes therefore too high to allow nucleation in the vicinity of the filler.

At last, the crystallization rate (estimated from the extrapolated slope defined above) of the different samples is reported in Fig. 9. The presence of a critical effective crosslink density for crystallization rate is confirmed. Thanks to additional data obtained from filled samples, our present description of the SIC phenomenon is refined compared with the previous one describing only the unfilled sample. Two different regimes, at weak and high effective crosslink density, were highlighted and the molecular weight between physical entanglements in natural rubber $\left(M_{\mathrm{e}} \sim 7000 \mathrm{~g} \mathrm{~mol}^{-1}\right.$ and $\nu_{\mathrm{e}} \sim 1.3 \times$ $10^{-4} \mathrm{~mol} \mathrm{~cm}^{-3}$ ) seems to correspond to the boundary between both regimes. Below the critical value $\left(\nu_{\mathrm{e}}\right)$, when a sample is stretched, nuclei number increases and the medium mobility decreases with the increase of effective crosslink density. Even if the growth step is slightly hindered, the nucleation step is so (a)

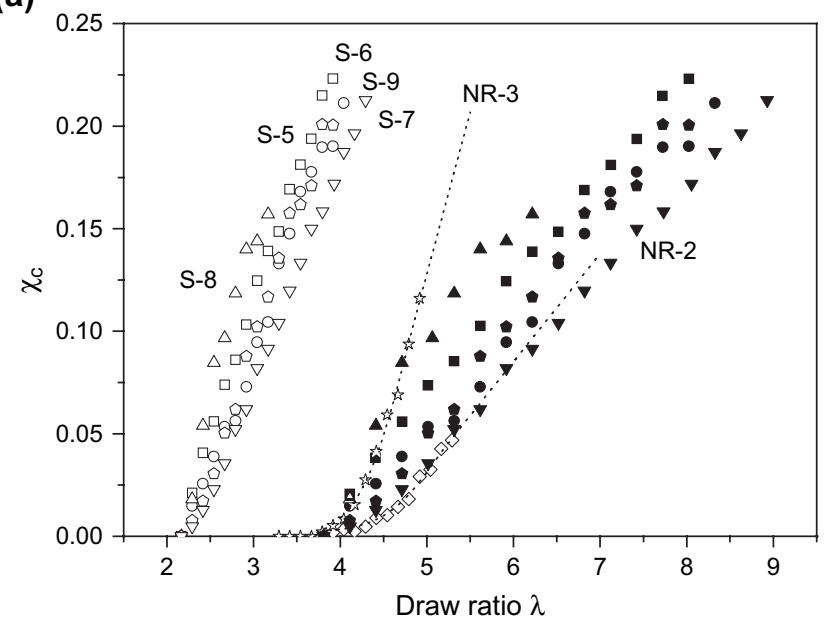

(b)

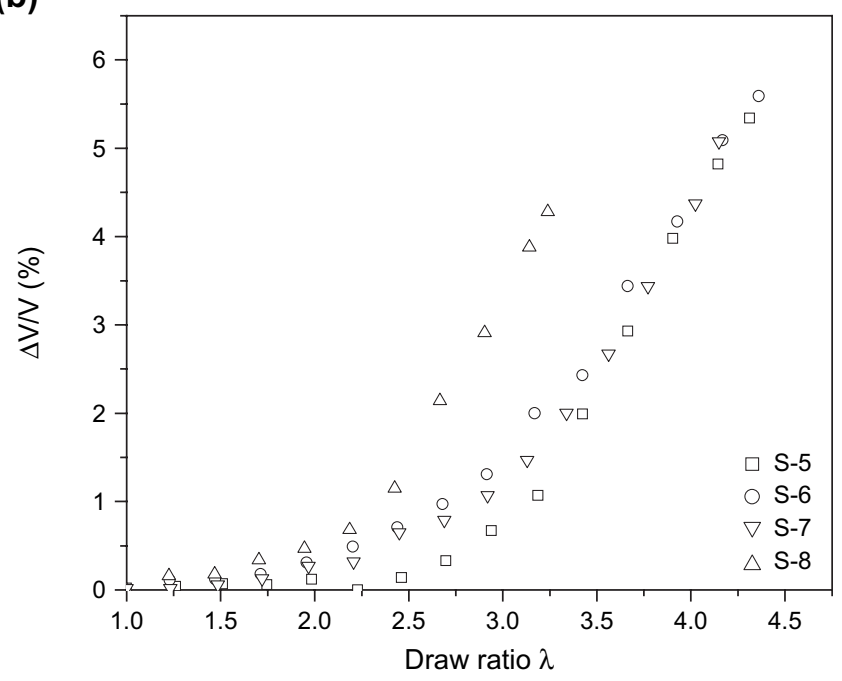

Fig. 11. (a) Effect of silica grafting and matrix density on samples' crystallization (open symbols). Crystallinity versus NR filled matrix local draw ratio deduced from Nielsen equation (closed symbols). (b) Volume change measurement of silica filled NR (S-5, S-6, S-7, S-8).

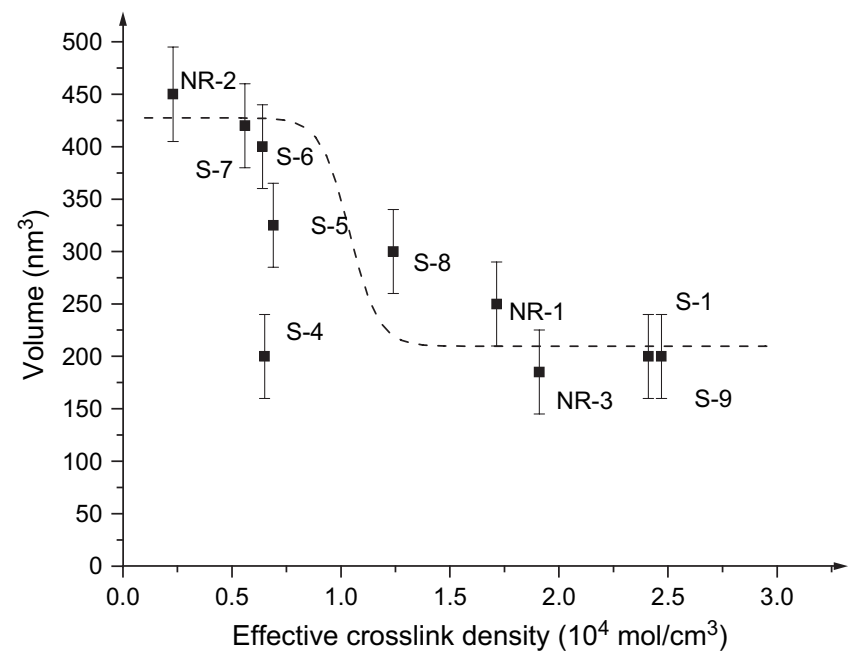

Fig. 12. Variation of average crystallites' volume versus effective crosslink density. 
promoted that the crystallization rate increases. Simultaneously, the crystallites' size slowly decreases until $\nu_{\mathrm{e}}$ where it strongly drops. Above the critical value, the restriction of the medium mobility becomes significant and hinders not only the growth but also the nucleation step. Thus, the crystallization rate slows down. The origin of this decrease of the nucleation rate can be related to the creation of an excluded volume in the filler vicinity. In this area, the crystallization is inhibited because of a reduced mobility.

Data extracted from the literature enable us to corroborate our hypothesis on the predominant role of the effective crosslink density. Poompradub et al. [7] studied 40 and $60 \mathrm{phr}$ calcium carbonate filled NR. The chemical crosslink density of NR matrix is higher than the critical value $\left(\nu_{\mathrm{e}}\right)$ and as it was expected, the higher the filler ratio i.e. the effective crosslink density, the slower the crystallization rate. Even if a lower volume fraction is not of industrial interest, complementary experiments performed with the NR-2 matrix filled with a lower filler volume fraction would be a nice way to definitively confirm our interpretation.

\section{Conclusions}

Synchrotron WAXD during stress-strain measurements have been performed on NR with different crosslink densities, unfilled or filled with nanoscopic silica and carbon black. From these measurements, pertinent information on crystallization onset and rate and crystallite size have been obtained. First, it was observed that Mullins effect decreases the intensity of the SIC. Mullins effect being highly dependent on the mechanical history of the material, it is necessary to "demullinize" the samples to study the influence of the material parameters on SIC. Secondly, it appears that the filler effect on SIC mainly depends on the NR matrix chemical crosslink density and filler loading. Depending on NR matrix chemical crosslink density, fillers (CB and silica) either accelerate or slow down the crystallization rate. Thus, the effective crosslink density can be defined and coherent description of SIC can be proposed taking into account the evolution of crystallization rate and crystallite size versus this parameter.

In the future, it will be interesting to prove if our conclusions about SIC of NR could be generalized to other polymers. In fact, strain induced crystallization is a common phenomenon to numerous vulcanized rubbers such as polyisobutylene, cis-1,4-polybutadiene... (except SBR). To our knowledge, the
SIC mechanism seems to be the same regardless of rubber type. However, a next study based on rubbers with very different molecular weights between entanglements $\left(M_{\mathrm{e}}\right)$ will enable to conclude about the universality of our present results.

\section{Acknowledgements}

We acknowledge the European Synchrotron Radiation Facility for provision of synchrotron radiation facilities and we would like to thank Dr. C. Rochas for assistance in using beamline D2AM. We thank Mr. B. Moreaux and Mme M.D. Charrier for preparation of samples.

\section{References}

[1] Kraus G. Reinforcement of elastomers. New York: Interscience Publication; 1965.

[2] Gent AN. Engineering with rubber. Oxford, England: Hanser Publisher, Oxford University Press; 1992.

[3] Allen PW, Jones KP. Natural rubber science and technology. Oxford: Oxford University Press; 1988.

[4] Lee DJ, Donovan JA. Rubber Chem Technol 1987;60:910-23.

[5] Trabelsi S, Albouy PA, Rault J. Macromolecules 2002;35:10054-61.

[6] Trabelsi S, Albouy PA, Rault J. Macromolecules 2003;36:9093-9.

[7] Poompradub S, Tosaka M, Kohjiya S, Ikeda Y, Toki S, Sics I, et al. J Appl Phys 2005;97:103529.

[8] Rault J, Marchal J, Judeinstein P, Albouy PA. Macromolecules 2006;39: 8356-68.

[9] Gehman SD, Field JE. Ind Eng Chem 1940;32:1401-7.

[10] Chenal JM, Chazeau L, Bomal Y, Gauthier C. Polymer 2007;48:1042-6.

[11] Ramier J, Chazeau L, Gauthier C, Ladouce L, Bouchereau MN. J Polym Sci Part B Polym Phys 2005;44:143-52.

[12] Sanders JF, Ferry JD, Valentine RH. J Polym Sci A-2 1968;6:967-80.

[13] Flory PJ, Rehner J. J Chem Phys 1943;11:512-20.

[14] Bandrup J, Immergut E, Grulke E, editors. Polymer handbook. New York: Wiley Interscience Publication; 1999.

[15] Wolf S. Rubber Chem Technol 1996;69:325-46.

[16] Sajjayanukul T, Saeoui P, Sirisinha C. J Appl Polym Sci 2005;97:2197203.

[17] Ramier J, Gauthier C, Chazeau L, Stelandre L, Guy L. J Polym Sci Part B Polym Phys 2007;45:286-98.

[18] Göritz D. Angew Makromol Chem 1992;202:309-29.

[19] Benedetti E, Corradini P, Pedone C. Eur Polym J 1975;11:585-7.

[20] Mitchell GR. Polymer 1984;25:1562-72.

[21] Boonstra BB. Polymer 1979;20:691-704.

[22] Nielsen LE. J Appl Polym Sci 1966;10:97-103

[23] Gent AN. Trans Faraday Soc 1954;50:521-33.

[24] Chenal JM, Chazeau L, Bomal Y, Gauthier C. J Polym Sci 2007;45: 955-62. 